\title{
Recognition of Anthracnose Injuries on Apple Surfaces using YOLOV 3- Dense
}

\author{
Mr. Rahul Sharma \\ Auricle Technologies Bikaner Rajasthan India \\ rahulmnul@gmail.com
}

\begin{tabular}{|l|l|}
\hline $\begin{array}{l}\text { Article History } \\
\text { Article Submission } \\
10 \text { February } 2015 \\
\text { Revised Submission } \\
27 \text { April } 2015 \\
\text { Article Accepted } \\
10 \text { May 2015 } \\
\text { Article Published } \\
30 \text { June 2015 }\end{array}$ & $\begin{array}{l}\text { Abstract } \\
\text { Plant ailment is one of the essential drivers of harvest yield decrease. With the } \\
\text { of plant surface sore pictures gathered by optical sensors has become a significant } \\
\text { research bearing for convenient yield ailment analysis. Right now, anthracnose } \\
\text { injury identification strategy dependent on profound learning is proposed. Right off } \\
\text { the bat, for the issue of lacking picture information brought about by the irregular } \\
\text { event of apple illnesses, notwithstanding conventional picture expansion strategies, } \\
\text { Cycle-Consistent Adversarial Network (CycleGAN) profound learning model is } \\
\text { utilized right now achieve information increase. These strategies adequately } \\
\text { enhance the decent variety of preparing information and give a strong } \\
\text { establishment to preparing the identification model. Right now, the premise of } \\
\text { picture information increase, thickly associated neural system (DenseNet) is used to } \\
\text { streamline highlight layers of the YOLO-V3 model which have lower goals. } \\
\text { DenseNet extraordinarily improves the usage of highlights in the neural system } \\
\text { and upgrades the identification consequence of the YOLO-V3 model. It is checked } \\
\text { in tests that the improved model surpasses Faster R-CNN with VGG16 NET, the } \\
\text { first YOLO-V3 model, and other three cutting edge arranges in discovery } \\
\text { execution, and it can understand continuous recognition. The proposed technique } \\
\text { can be all around applied to the recognition of anthracnose injuries on apple } \\
\text { surfaces in-plantations. } \\
\text { Keywords: CycleGAN, R-CNN, YOLO-V3 }\end{array}$ \\
\hline
\end{tabular}

\section{Introduction}

Agriculture is the basic need of every human in this world. India is an agricultural based country $70 \%$ of the Indians are either directly or indirectly involved in agricultural works. Indian economy depends on the production of agricultural products. The growing countries like India occupied with large population with insufficient food supply and food production. This is due to various factors like urbanization, colonization and industrialization. The people started moving towards cosmopolitan cities besides their native villages. This causes the poor improvement of Agricultural and farming Technologies. Failures of monsoon, unavailability of water, improvement of concrete jungles are the major factors that act as desolation of cultivable lands. The poor knowledge of farmers about agronomy leads less production of grains [1][2].

The continuous industrialization and unemployment problem provoke the poor settlement of agriculture technology [3]. Due to population explosion we have to adopt intensive methods to improve crop production. The existing techniques lead to green revolution. At the same time production methods also decreased due to the nonavailability of advanced technology which is required in the need for good agricultural practice. Therefore, we have to follow certain productivity- based technology in the improvement of crop production (Changmai 2018). The existing technologies such as nanotechnology, microbiology and biotechnology tried its best outcome in the 
field of agriculture and horticulture. But it's not saturating the level according to the increasing of population. Natural calamities play a vital role in the production of crops, monsoon failure, global warming and Ozone [4].

A farmer can sustain if he sells the products that the produced with a profitable price. If any ailment is present in the fruits the buyers will resist to buy and this will cause a huge loss. With this instinct we have designed such project which will give the solution to rectify the problem to identify the diseased apples in early stage and sell it or remove from that place so that it will not be speeded [5].

The primary reason for crop yield reduction is due to plant ailment. To reduce these kind of economic losses in agriculture, control measures have taken like accurate discernment of disease and timely detection of plant disease. It is hard to find lesions in branches, leaves, blossoms and natural products in time because of planting zone and crown stature of fruit trees. Moreover, the information and experience of organic product grower differ extraordinarily, which may prompt confusion of infection types and incorrect estimation. Plant infections cannot be controlled because inappropriate cultivating choices bring about the misuse of work and assets. Normally, diseased plant is in sharp contrast with healthy plant where the diseases alter the surface of plant branches, leaves and fruits [6].

In real-time now, only the diseased apples can be detected one by one using YOLO. It cannot detect in orchards so that after plucking from the trees the apples cannot be sold that will cause a huge loss to the fruit farmers.

Here we combined CycleGAN and YOLO-V3 for the betterment of results. All the apples with all available shapes cannot be found as both healthy and diseased. For that CycleGAN will be the solution for creating the data set. The newest version of YOLO which is YOLO-V3 that takes 40 frames/sec is the fastest way of detecting small objects [7].

\section{Related Work}

Paustianet al proposed that the attention in precision farming has been increasing in recent years. Farmers are able to manage the temporal crop, soil variability and spatial within a field to reduce costs, increase quality, profitability, and optimize yield are provided by precision farming, which is an integrated approach system. In the precision agriculture technologies adopted by the farmers there has been a significant research. In German crop farming, in order to gain perception into the apt features of precision farming, a vast range of farmer demographics and characteristics of farm are tested. For the predictors having positive influence on the perception of precision farming ,the results of a logistic regression analysis show that they are services of agricultural contractor that is farming business in addition, having experience under 5 years or 16 and 20 years in crop farming, and having arable land of more than 500 ha. Anyhow, producing barley with farm less than 100 ha are factors that employ an influence of negativity on the precision farming perception[8].

Yuanshen Zhao et al proposed that the commercial application of robots used in harvesting has been limited due to the deficiency in robust fruit recognition access and capabilities of precision picking ,eventhough there is a lightning development in technologies of agricultural robots. On the other hand, this situation has been improved by the advances in vision-based control techniques. These techniques include methods of eye-hand coordination, algorithms of recognition of fruit and strategies of vision information acquisition. In vegetable or fruit harvesting robot, using visual information, two major problems has been solved in object detection in object picking and tree canopies by employing vision control. This presented a review on potential applications in vegetable or fruit harvesting robots.

Wang et al proposed that in the management of orchards of apple estimation of crop yield is a vital task. At present the sampling is done manually which is not accurate, consuming time and more 5 labors required. To deal with this, a system based on computer-vision is developed for the yield estimation to be automated and accurate. The system consists of 2 cameras which work in night time and to reduce the natural illumination variance artificial lighting is attached. For automatically collecting the data an automatic orchard vehicle is used. The system 
captures image and counts the apples for crop yield estimation. The system is tested with both red and green apples. For 480 trees of red apples the error is $-3.2 \%$ and for about 670 trees of green apples the error is $1.2 \%$.

Sankaran et al proposed that worldwide the major production losses and economic losses in agriculture is due to plant diseases. For imperishable agriculture, disease detection and health monitoring of trees and plants was not easy. For the real-time monitoring of tree health condition there is no commercial sensors available. A costeffective, expeditious, and dependable sensor that monitors plant and tree health that would ease evolution in agriculture was recognized as a need. The current technologies can be utilized for ground-based sensor system development to aid in observing health of the plants and diseases in plants. These include technologies like based on image and spectroscopic, detection of plant diseases based on volatile profiling [9].

Salathéet al proposed that due to the absence of mandatory infrastructure, in many parts of the world the crop disease is not identified rapidly so that in food security crop disease is a major threat. The smartphone-assisted disease diagnosis is possible due to deep learning which lead to the combination of recent advancements in computer vision and global smartphone perforation increment. In order to find 26 diseases and 14 crop species a deep 7. convolutional neural network is trained using dataset of 54,306 images of healthy and diseased plant leaves are collected under controlled scenario. Indicating the practicability of the approach $99.35 \%$ accuracy has been achieved by this trained model. A path to diagnosis of crop disease using smart phone has cleared by the training of images that available publicly and increasingly large image data sets using deep learning [10].

Ferentinos et alproposed that using methodologies of deep learning, to perform detection of plant diseases and diagnosis using images of leaves of diseased and healthy plants a convolutional neural network has been developed. From 58 different classes, in 25 distinct plants that include both healthy and diseased plants a database of 87,848 images have been collected for the training the model. The success rate of $99.53 \%$ in identifying correct combination has been achieved by training the model architectures. Due to its high success rate it can be expanded to detect fused plant disease to help real cultivation conditions.

Redmon et al proposed that YOLO-V3 is a fine detector. It is accurate and fast. The COCO Average Precision (AP) is between .5 and .95 in Intersection Over Union (IOU) metric, YOLO-V3 is not greater than that. In the former detection metric of .5 Intersection Over Union, YOLO-V3 is good. In target detection YOLO-V3 directly uses regression instead of adopting region proposal procedure. So that, the process of detection is accelerated effectively. The present version YOLO-V3 has a tremendous performance in detecting small objects rather than high speed and desirable precision. That's why YOLO-V3 is an opulent reference for detecting apple lesions. Even so, it has not been used for detecting plant lesions widely.

\section{Recognition of Anthracnose Injuries on Apple Surfaces}

Anthracnose is one of the most important disease of apple fruit caused by fungus Neofabraeamalicorticis. As it ripens and become mummified the fruits get rots quickly and that may remain attached to the tree throughout the season. Large number of spores are produced under humid conditions and the spore mass appear crystalline under dry conditions. The decayed tissue underneath the outside of the sore is watery, showing up in a V-molded example in cross segment that limits toward the centre. They attack the plant tissues forcefully, killing the host cells to get nutrients. During winter season, the pathogen get dormant and lesion progression ceases. Fungal spores appear on it to infect healthy fruit and bark.

To avoid this remove fruits from orchards and keep dry after harvest. Store it in low oxygen atmosphere.The translation of image-to-image includes generation of new version of an input image with given modifications, like translation of winter landscape to summer. In image-to-image translation model training, the paired example data sets must be large. The preparation of datasets is not so easy and also expensive, and in certain instances it is impossible, like painting photographs by dead artists.For dealing with this kind of problems the translations must be "Cycle Consistent". 


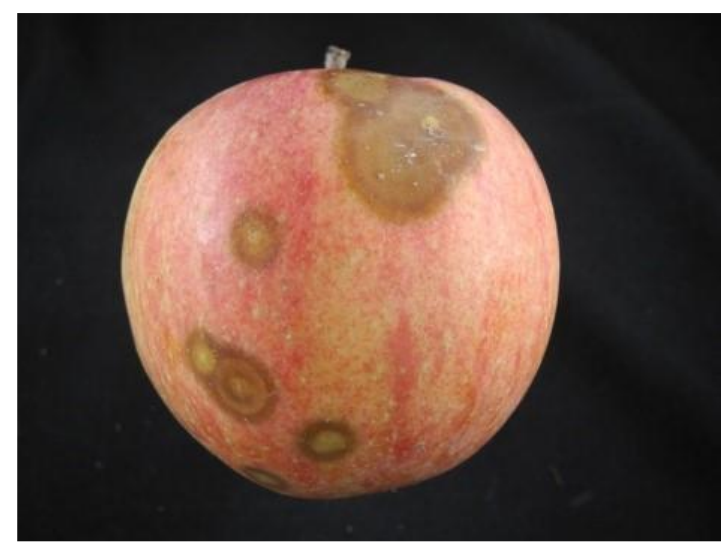

Fig.1 Diseased apple

Example: if we translate English sentence to Spanish sentence and again translate that Spanish sentence to English sentence, the original sentence must be acquired. Likewise if an image $\mathrm{X}$ is translated to $\mathrm{Y}$ with $\mathrm{G}$ mapping and that $\mathrm{G}(\mathrm{X})$ is again translated to $\mathrm{X}$ using $\mathrm{F}$ mapping original image should be arrived. Such that, CycleGAN has two networks of GAN, a generator network and a discriminator network. One cycle consistency loss and two adversarial losses are used for training the network.

Magnificent results have been achieved in representation learning, generation of an image and editing of an image using GAN(Generative Adversarial Network). Adversarial loss is the main advantage in GAN that makes the images to be indistinct from the real images. Much of the computer graphics optimization is based on this loss that is used for generation of images tasks powerfully.

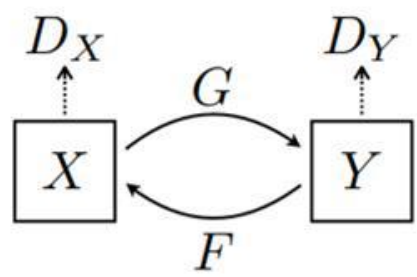

(a)

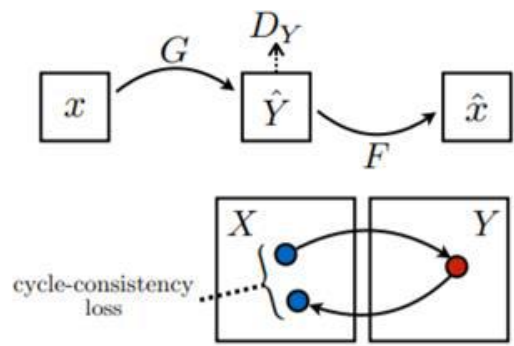

(b)

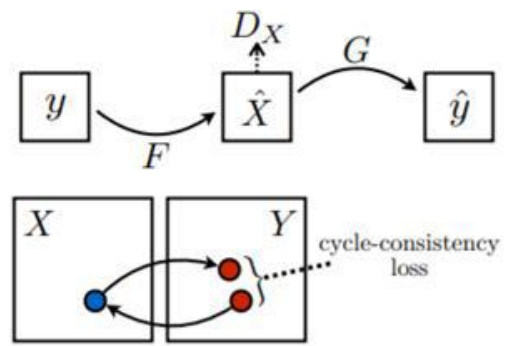

(c)

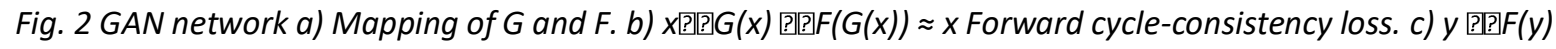
[D] $G(F(y)) \approx y$ backward cycle consistency loss.

The generator consists of three sections namely: Encoder, Transformer and Decoder. Encoder: It consists of three convolutional layers. The input image is first passed through the encoder and it extracts the main features of the image. Transformer: It consists of six blocks of residue, which takes the main features that are extracted from the encoder as input and provides the output. Decoder: In the decoder de-convolution takes place and the transformer output is processed into a new image.

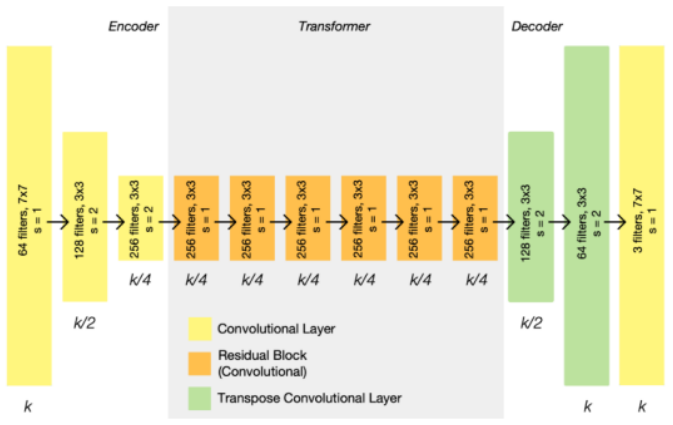

Fig. 3 Generator Architecture 
Adversarial loss for G is DX and for F is DY. For mapping correctly from input to output cycle consistency loss is also needed. The full loss is as follows:

$$
\mathrm{L}(\mathrm{G}, \mathrm{F}, \mathrm{DX}, \mathrm{DY})=\operatorname{LGAN}(\mathrm{G}, \mathrm{DY}, \mathrm{X}, \mathrm{Y})+\operatorname{LGAN}(\mathrm{F}, \mathrm{DX}, \mathrm{X}, \mathrm{Y})+\lambda \operatorname{Lcyc}(\text { FmG })
$$

where LGAN( G, DY, X, Y) is the loss of G and DY, LGAN (F, DX, X, Y) is the loss of F and DX, and Lcyc F, $\mathrm{G}$ is the cycle consistency loss. The expectation of Cycle GAN model is as follows:

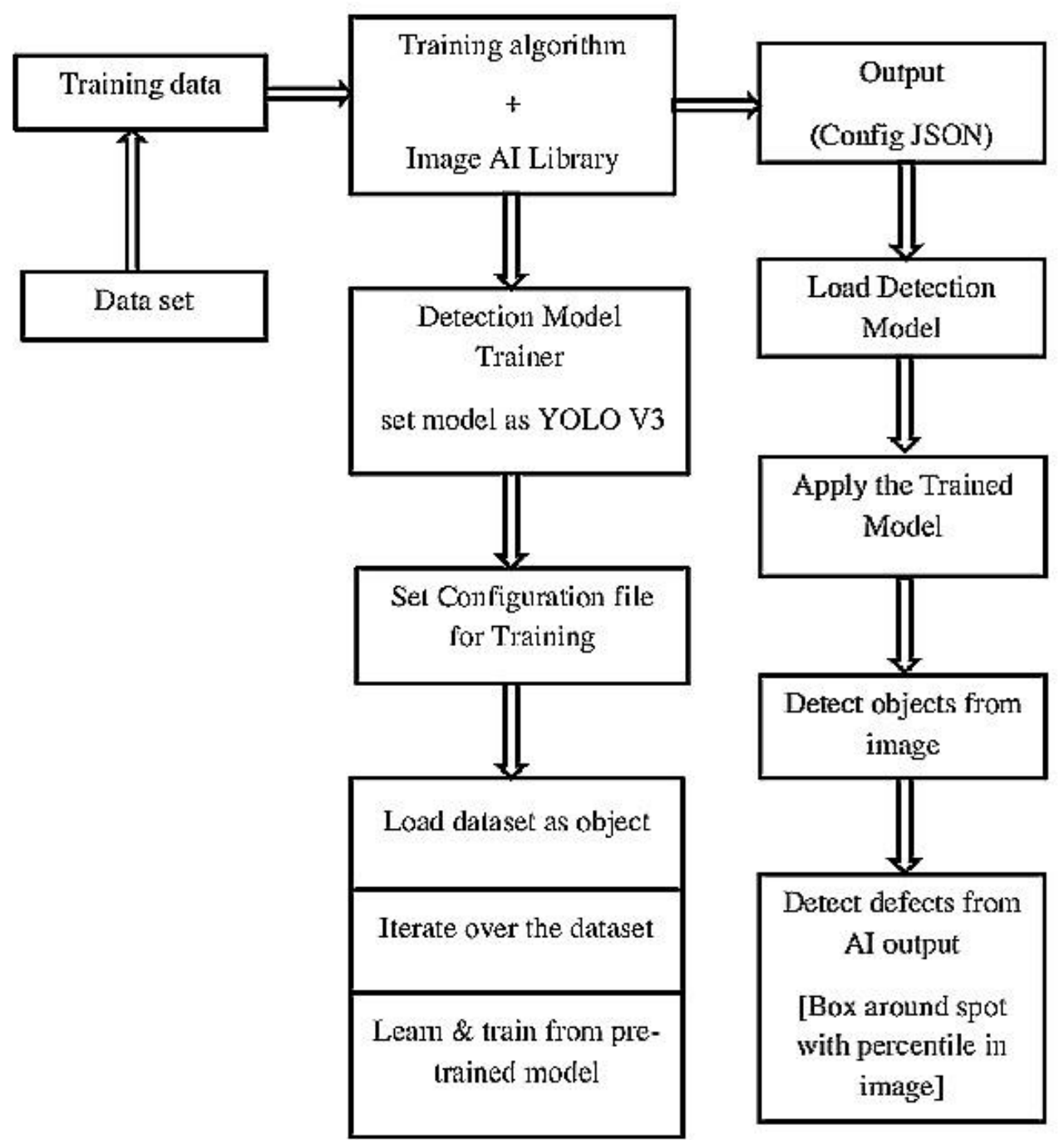

Fig:4 Flowchart representation of working model

The feature usage efficiency can be improved by DenseNet. The input images are scaled to $512 * 512$ pixels. The lowest resolution of down-sampling layers are replaced with DenseNet. The dataset is created using CycleGAN and trained to detect diseased apple. The algorithm is used from Image AI library. The process is done in google collaborative notebook which is used for machine learning projects, it has both anaconda and jupyter platforms, here jupyter is used. For detection of defected apples YOLO V3 is used from the library, which creates a box around the diseased apple with percentile. 


\section{Simulation Results}

Tensor Flow is one of the open source software library to perform numerical computations and tasks to develop deep learning models. It is one of the cross-platform works in everything like GPU, CPU including mobile and embedded platforms. Google collaborator is used to develop applications of deep learning using Keras, TensorFlow, PyTorch and OpenCV. Google Colab is free cloud service and it is used to support GPU. It is important to note that one could improve the python coding skills. Image AI is one of the Python library, it is used to perform object detection on images and to extract information from images.

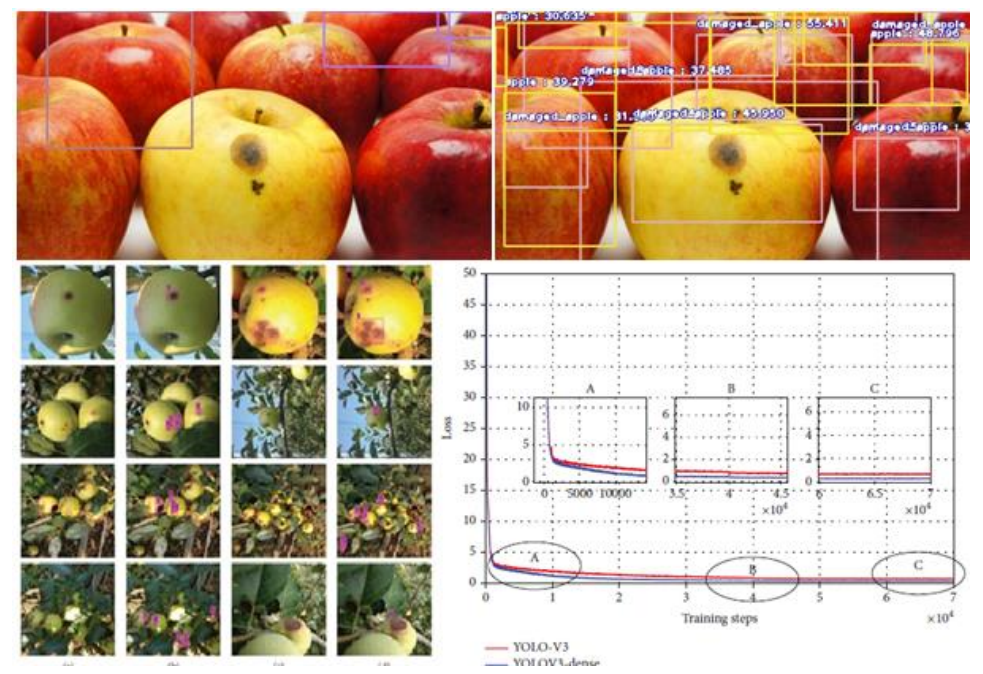

Fig. 5 Detection of diseased apple and comparison with the existing model

\section{Conclusion}

The anthracnose injury location strategy is proposed dependent on profound learning. Right off the bat, taking into account the issue of deficient picture dataset because of the arbitrary event of apple sicknesses, CycleGAN profound learning technique is received to extricate the highlights of solid apples and anthracnose apples and to create anthracnose injuries on the outside of sound apple pictures. Fruit farming is one of the main field in farming, and the farmers are doing hard work in the field to grow it. If the profit is not up to the level their entire work will be waste. So in order to prevent such situation this project will be helpful in detecting diseased using YOLO V3 apples in orchards such that it can be sold earlier or removed from the place to prevent spreading. The project can be extended for detecting many fruits in single capture. The real time application can be made using mobile, Ipad or any other camera devices.

\section{References}

[1] Z. Xiao-bo, Z. Jie-wen, L. Yanxiao, and M. Holmes, "In-line detection of apple defects using three colour cameras system," Computers and Electronics in Agriculture, vol. 70, no. 1, pp. 129-134, 2010.

[2] J. Hartman, -Apple Fruit Diseases Appearing at Harvestll, Plant Pathology Fact Sheet, College of Agriculture, University of Kentucky, http://www.ca.uky.edu/agcollege/plantpathology/ext_files/PPFShtml/PPFS-FR-T-2.pdf, viewed on December 2011.

[3] Q. Li, M. Wang, and W. Gu, -Computer vision based system for apple surface defect detection,\| Computers and Electronics in Agriculture, vol. 36, pp. 215-223, Nov. 2002.

[4] P. M. Mehl, K. Chao, M. Kim, and Y. R. Chen, -Detection of defects on selected apple cultivars using hyperspectral and multispectral image analysis, $\|$ Applied Engineering in Agriculture, vol. 18, pp. 219-226, 2002. 
[5] M. S. Kim, A. M. Lefcourt, Y. R. Chen, and Y. Tao, -Automated detection of fecal contamination of apples based on multispectral fluorescence image fusion,॥ Journal of food engineering, vol. 71, pp. $85-91,2005$.

[6] O. Kleynen, V. Leemans, and M. F. Destain, -Development of a multi-spectral vision system for the detection of defects on apples, $\|$ Journal of Food Engineering, vol. 69, pp. 41-49, 2005

[7] Dubey, Shiv Ram \& Jalal, Anand. (2012). Detection and Classification of Apple Fruit Diseases Using Complete Local Binary Patterns. Proceedings of the 2012 3rd International Conference on Computer and Communication Technology, ICCCT 2012. 10.1109/ICCCT.2012.76.

[8] Dubey, Shiv \& Jalal, Anand. (2012). Adapted Approach for Fruit Disease Identification using Images. International Journal of Computer Vision and Image Processing. 2. 51-65. 10.4018/ijcvip.2012070104. 